\title{
Machine Learning and Internet of Medical Things for Handling COVID-19: Meta-Analysis
}

\author{
Shahab S. Band ${ }^{1 *}$, Sina Ardabili², Atefeh Yarahmadi' ${ }^{1}$, Bahareh Pahlevanzadeh ${ }^{3}$, Adiqa Kausar \\ Kiani $^{1}$, Amin Beheshti ${ }^{4}$, Hamid Alinejad-Rokny ${ }^{5,6,7}$, Iman Dehzangi ${ }^{8,9}$, Amir Mosavi ${ }^{10 *}$ \\ ${ }^{1}$ Future Technology Research Center, College of Future, National Yunlin University of Science and \\ Technology, 123 University Road, Section 3, Douliou, Yunlin 64002, Taiwan \\ ${ }^{2}$ Department of Informatics, J. Selye University, Komarom, Slovakia \\ ${ }^{3}$ Department of Design and System Operations, Regional Information Center for Science and \\ Technology (RICEST), Shiraz, Fars, Iran \\ ${ }^{4}$ Department of Computing, Macquarie University, Sydney 2109, AU \\ ${ }^{5}$ BioMedical Machine Learning Lab, The Graduate School of Biomedical Engineering, UNSW \\ Sydney, Sydney, NSW, 2052,, Australia \\ ${ }^{6}$ UNSW Data Science Hub, The University of New South Wales (UNSW Sydney), Sydney, NSW, \\ 2052, Australia \\ ${ }^{7}$ Health Data Analytics Program, AI-enabled Processes (AIP) Research Centre, Macquarie \\ University, Sydney, 2109, Australia \\ ${ }^{8}$ Department of Computer Science, Rutgers University, Camden, NJ, 08102, USA \\ ${ }^{9}$ Center for Computational and Integrative Biology, Rutgers University, Camden, NJ, 08102, USA \\ ${ }^{10}$ John von Neumann Faculty of Informatics, Obuda University, Budapest, Hungary
}

\section{* Correspondence:}

Shahab S. Band, Amir Mosavi

shamshirbands@yuntech.edu.tw; amir.mosavi@kvk.uni-obuda.hu

Keywords: Machine Learning, COVID-19, Internet of Things (IoT), Deep Learning, Big Data

\begin{abstract}
Early diagnosis, prioritization, screening, clustering and tracking of COVID-19 patients, and production of drugs and vaccines are some of the applications that have made it necessary to use a new style of technology to involve, to manage and deal with this epidemic. Strategies backed by artificial intelligence (AI) and the Internet of Things (IoT) have been undeniable to understand how the virus works and try to prevent it from spreading. Accordingly, the main aim of this survey article is to highlight the methods of ML, IoT and the integration of IoT and ML-based techniques in the applications related to COVID-19 from the diagnosis of the disease to the prediction of its outbreak. According to the main findings, IoT provided a prompt and efficient approach of following the disease spread. Most of the studies developed by ML-based techniques for handling COVID-19 based dataset provided performance criteria. The most popular performance criteria, is related to accuracy factor. It can be employed for comparing the ML-based methods with different datasets. According to the results, CNN with SVM classifier, Genetic CNN and pre-trained CNN followed by ResNet, provided
\end{abstract}


highest accuracy values. On the other hand, the lowest accuracy was related to single CNN followed by XGboost and KNN methods.

\section{Introduction}

The COVID-19 outbreak has created many challenges in human life around the world [1,2]. The most devastating impact which is increasing casualties and deaths (around the world) have made it clear the need for social and business restrictions [3]. With the expansion of the COVID-19 pandemic, the world community has faced many other problems in various aspects of life such as, economic and social life, psychological wellness, political interactions, cultural activities, educational limitations, religious restrictions, and even sport events $[4,5]$. Such examples highlight the need for effective and intelligent systems to deal with such crises in pandemic situation [5]. Early diagnosis, prioritization, screening, clustering and tracking of patients, and production of drugs and vaccines are some of the applications that have made it necessary to use a new style of technology to involve, to manage and to deal with this epidemic [6]. Machine Learning (ML) and Artificial intelligence (AI) algorithms displayed promising ability in prediction and classification [7-18] including disease prediction [19-30], virus genome analysis [20,31,32], and medical imaging and Internet of Things [33-36]. Strategies backed by artificial intelligence (AI) and the Internet of Things (IoT) have been undeniable to understand how the virus works and try to prevent it from spreading [5, 37]. These techniques have evolved with the development of computing resources with cloud computing and recent advances in machine learning (ML). All of these advances enable researchers to successfully process large amounts of data and extract information from it. ML-based methods used in processing and modeling data on COVID-19 disease can increase efficiency and speed up results by improving computations.

Gou et al. developed a survey for evaluating the ML-based techniques for diagnosis of COVID-19 using medical data collection, image preprocessing, feature extraction, and image classification. The study evaluates Transfer, ensemble, unsupervised and semi-supervised learnings, and convolutional neural networks, graph neural networks, and explainable deep neural networks. Evaluations focused on the advantages and limitations of the diagnosis techniques [38]. Abumalloh et al. presented a stateof-the-art of ML-based techniques for handling medical image processing in the context of the COVID19 crisis [39]. Khan et al. developed a survey of the applications of AI for preventing the COVID-19 pandemic [40].

As can be deduced, many survey studies have been developed in this regard. But, the existence of a study that can systematically review and discuss two interrelated areas of the ML and the IoT in the form of an article has been lost from the research literature. Accordingly, the main purpose of this review article is to examine the methods of ML, IoT and the integration of IoT and ML-based techniques in the applications related to COVID-19 from the diagnosis of the disease to the prediction of its outbreak.

The study has three main sections. The first section is to present the importance of IoT and IoT-ML based techniques in COVID-19 applications. The second section is to present the importance of MLbased techniques in COVID-19 applications. The last part is to present the main findings, challenges and future perspectives.

\section{Methodology}

\subsection{Dataset preparation}


Systematic review may successfully provide technical and effective literature for a specific topic [41]. A systematic review requires proper collection of papers around the subject. Preparing dataset is one of the main steps of determining the quality of review work [41]. Finding the most related studies requires proper keywords and proper searching libraries. In the present study, the most relevant studies have been collected from WOS, and Scopus libraries. Table 1 presents the main keywords and searching queries.

Table 1. Searching queries

\begin{tabular}{lll}
\hline Search within & Operators & keywords \\
\hline Article title, Abstract, Keywords & AND & COVID-19, Pandemic, diagnosis, detection \\
Abstract, Keywords & OR/AND & COVID-19, Pandemic, Prediction, Monitoring \\
Abstract, Keywords & OR/AND & COVID-19, Pandemic, Classification, Identification \\
Abstract, Keywords & OR/AND & COVID-19, Pandemic, IoT, Machine learning \\
\hline
\end{tabular}

\subsection{IoT for COVID-19}

IoT is an interconnected set of computing tools from simple to complex that can be used in conjunction with mechanical or digital machines in the presence of humans, animals, or objects. IoT technology can easily transfer data from the source to the destination through the network without the presence of the operator. This technology can actually be considered as a special tool in human-human interaction or human-computer interaction $[42,43]$. An IoT platform includes the minimum equipment required, such as smart devices equipped with the web [44]. These systems consist of processors, sensors, and communication hardware to collect, send, control, manage, and generally convert data into accessible data $[44,45]$. These systems connect to an IoT port used to send data to the cloud so that data can be analyzed and shared [46]. These devices can operate by connecting to other related systems based on the information they receive [47]. These tools perform many of their tasks without human intervention.

Today, the use of IoT technology in the field of health and treatment is growing, rapidly [48]. The main applications of IoT in the process of treatment and intelligent health can include identification, digitization of medical information, patient transfer to the hospital, use of vital signs sensors, use of smart phones in communication, and in general, digitization of medical processes [49, 50]. Use of IoT has become more popular and important due to COVID-19 pandemic [51]. Due to the fact that this virus is highly contagious and has a high risk to human health and has caused many problems for the medical staff, using non-contact methods to diagnose as soon as possible, control patients, monitor the condition of patients with acute illness, as well as maintaining social distance, can be an important factor in breaking off part of the virus infection cycle $[52,53]$. In the field of non-contact methods, the IoT is a leader and can solve many problems in this field [54]. Figure 1 presents the main applications of the IoT in COVID-19 era. 


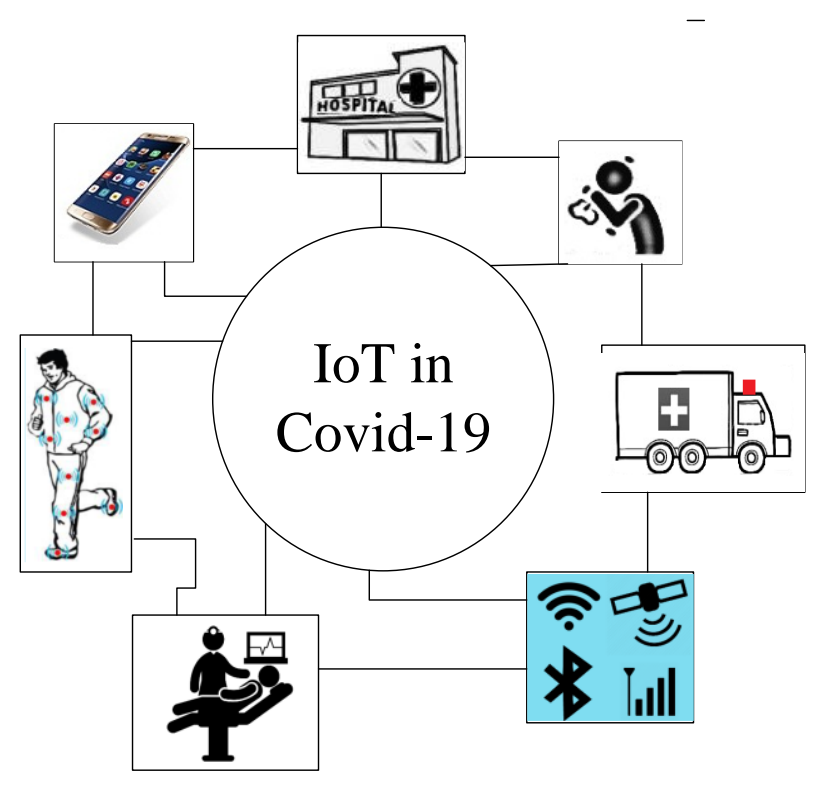

Figure 1. Applications of IoT in COVID-19

Table 2 presents the highlighted studies for the application of IoT-based techniques to tackle COVID19. This table represent the studies based on the main four columns. Objective that briefly describes the main objective of each study. Methodology/proposed algorithm that presents the main algorithm and procedure employed by each study. Keyword indicates the main points and concentration of the study and finally, application section presents the field of the application of each method.

Table 2. The main studies for the application of IoT based techniques for handling COVID-19

\begin{tabular}{|c|c|c|c|c|c|}
\hline Order & Objective & $\begin{array}{l}\text { Methodology/Proposed } \\
\text { Algorithms }\end{array}$ & Keywords & Application & Reference \\
\hline 1 & $\begin{array}{l}\text { To aim an innovative IoT-based } \\
\text { online solution for tracking } \\
\text { COVID-19 outbreaks }\end{array}$ & $\begin{array}{l}\text { IoT-based platform to contact } \\
\text { and to trace the infection }\end{array}$ & $\begin{array}{l}\text { IOT: symptom-based } \\
\text { device-to-device (D2D) } \\
\text { communication }\end{array}$ & $\begin{array}{l}\text { Prediction and } \\
\text { monitoring }\end{array}$ & {$[55]$} \\
\hline 2 & $\begin{array}{l}\text { To compare DL techniques to } \\
\text { detect COVID- } 19\end{array}$ & $\begin{array}{l}\text { DL-based COVID-19 diagnosis } \\
\text { technique in order to model } \\
\text { instances for each type and to } \\
\text { diagnosis the vulnerabilities }\end{array}$ & IOT: DL algorithm, AE & Diagnostic & {$[56]$} \\
\hline 3 & $\begin{array}{l}\text { To develop an IoT-based DL } \\
\text { platform for early detection of } \\
\text { COVID-19 }\end{array}$ & $\begin{array}{l}\text { Chest X-Ray pictures for } \\
\text { training and testing of Regional- } \\
\text { based Convolutional Neural } \\
\text { Networks (RCNN) through IoT- } \\
\text { based framework }\end{array}$ & $\begin{array}{l}\text { IoT, COVID-19, Deep } \\
\text { learning, Region Proposal } \\
\text { Network (RPN) }\end{array}$ & Diagnostic & {$[57]$} \\
\hline 4 & $\begin{array}{l}\text { To develop a monitoring and } \\
\text { detection system according to } \\
\text { real-time data from in the } \\
\text { presence of the machine } \\
\text { learning algorithms }\end{array}$ & $\begin{array}{l}\text { SVM, ANN, Naïve Bayes, K- } \\
\text { NN, DT, Decision Stump, 1-R, } \\
\text { and 0-R. }\end{array}$ & $\begin{array}{l}\text { machine learning } \\
\text { algorithms, COVID-19 }\end{array}$ & $\begin{array}{l}\text { Identification } \\
\text { and monitoring }\end{array}$ & {$[58]$} \\
\hline
\end{tabular}


To investigate the IoT for diagnosis of COVID-19 patients using interconnected network

To investigate participants' health conditions and remembering the maintain physical distancing

To aim a smart edge

7 monitoring system using smart gadgets

To employ a noncontact IR sensor to evaluate for the body temperature

To develop a Medical

9 Diagnosis Humanoid to provide a complete diagnostic system for COVID-19

To develop a low-cost robotic system to diagnosis and help virus affected people

To contribute IoT and associated sensor technologies to trace, track and mitigate COVID-19 virus by developing hardware sensor

To extract the social relationships between mobile devices by allocating the limited protective resources

To develop Internet of Things to prevent the spreading of COVID-19

To develop a platform for biometric face detection along with COVID-19 outbreaks

To introduce a high resolution AQ monitoring system

To develop an IoMT architecture with respect to combat COVID-19.

To test information technology pandemic

12 IoT based monitoring systems are identified and discussed.

Internet of things (IoT) Monitoring

A lightweight and low-cost IoT node using a smartphone, and fog-based ML for data handling

To diagnose coronavirus infection using gadgets, deep edge computing and IoT to detect the virus suspected $\mathrm{H} 2 \mathrm{H}$ chain

Internet of Things (IoT), smartphone application, Machine Learning (ML), Monitoring Fuzzy system

COVID-19, Edge Computing, IoT

Monitoring

Checking the health condition

IoT, detection system

Detection

Autonomous navigation, detection, and monitoring system

IoT, AI, ML, Medical Diagnosis Humanoid

Monitoring and Diagnosis

To track hand gestures using radio frequency

to integrate IoT techniques and provide insight on the expected outcomes

To employ dynamic WUG model using social IoT

investigating an infected person using IoT

IoT-based Multi-task Cascaded Convolutional Network

A preliminary validation of the Air Heritage pervasive Air Quality monitoring concept

IoMT platform, emerging IoMT applications, to apply within the medical environment

AI, block-chain, Big Data and robots, for optimally handling pandemics
Wireless Robot, Gesture Recognition, IoT

AI, IoT, big data, data sharing, cloud computing

Social Internet of Things; susceptible-exposedinfected-removed; reinforcement learning

Internet of Things; health Detecting and care; cloud computing Monitoring

detection, cascaded CNN, Detection and edge computing,

Smart Air Quality monitors, IoT, Artificial Intelligence, COVID19,

COVID-19,

IoMT application, Detection security

COVID-19, information technology, AI, big data, indonesia
Diagnosis and monitoring system

Diagnosis and monitoring system recognition

Monitoring

Detection and monitoring pandemic 


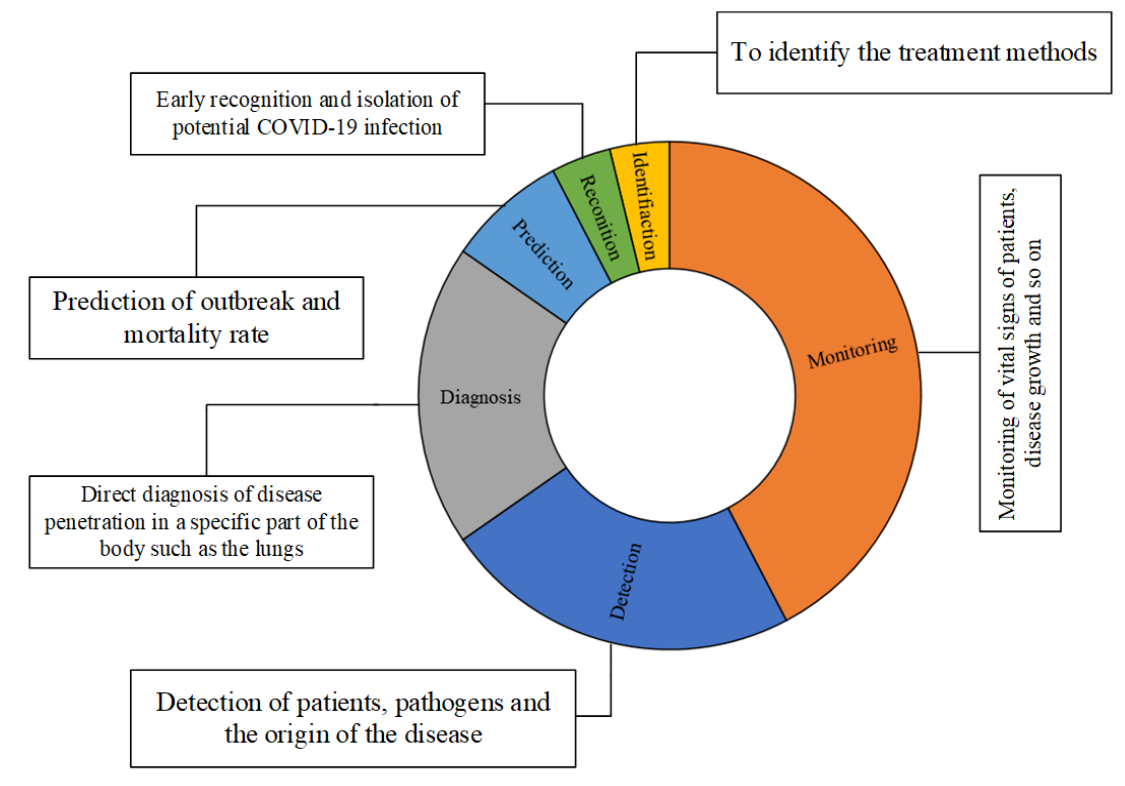

Figure 2. The share of each application type for IoT-based systems

Figure 2 is generated based on Table 2 to present the main applications and their portions by studies for handling IoT in COVID-19 pandemic. Monitoring, detection, and diagnosis are the main application of IoT based techniques in tackling COVID-19 pandemic. Monitoring can be performed in different ways. Accordingly, Roy et al. employed IoT as a real-time solution for monitoring of COVID-19 outbreaks [55]. Also Otoom et al employed IoT to provide monitoring and detection data using real-time system to feed to the machine learning algorithms for further applications or handling [58]. Singh et al and Vedaei et al used IoT as a tool for monitoring COVID-19 patients and their health condition in cooperating an interconnected network $[59,60]$. Ashraf et al proposed a smart edge surveillance system to monitor wearable smart gadgets which are operated according to IoT based technology [61]. Karmore et al developed a Medical Diagnosis Humanoid to provide a complete diagnostic system for COVID-19 using IoT based technology [63]. De Vito et al presented the outputs of a high resolution AQ monitoring system which was based on an IoT based technique [69].

Baskaran et al used a non-contact infrared sensor to examine for the body temperature for the detection of the COVID-19 patients [62]. Wang et al exploited the social relationships in the platform of Social IoT to solve controlling issues of COVID-19 epidemic by sharing the limited protective resources [66]. Kumar et al investigated an IoT based platform to prevent the spreading of COVID-19 [67]. Kolhar et al developed a platform of a decentralized IoT based biometric based on face detection platform for handling COVID-19 outbreaks [68]. Aman et al developed an architecture of IoT based framework for medical applications with respect to combat COVID-19 [34]. Manalu et al investigated the information technology to response COVID-19 pandemic trend in accordance with the IoT technology [70]. 
Figure 3 presents the main contribution of these papers. According to the reviewed studies, COVID19 dataset can be imported from three main sources including Radiography, statistics of health centers, and Sensors for prediction, monitoring, identification, detection, diagnosis and classification purposes. The output of the techniques needs to be evaluated for confirming the approach performance and accuracy values. The frequently used parameters for performance analyzing include Accuracy, Precision, Recall, RMSE, Correlation coefficient and mean absolute percentage error. This can be considered as a brief explanation as the main contribution of the present study. This study successfully presents the advantages and disadvantages of each technique for a specific task in handling COVID19 dataset and propose the future perspectives. Also, this study can detect the main challenges and limitations.

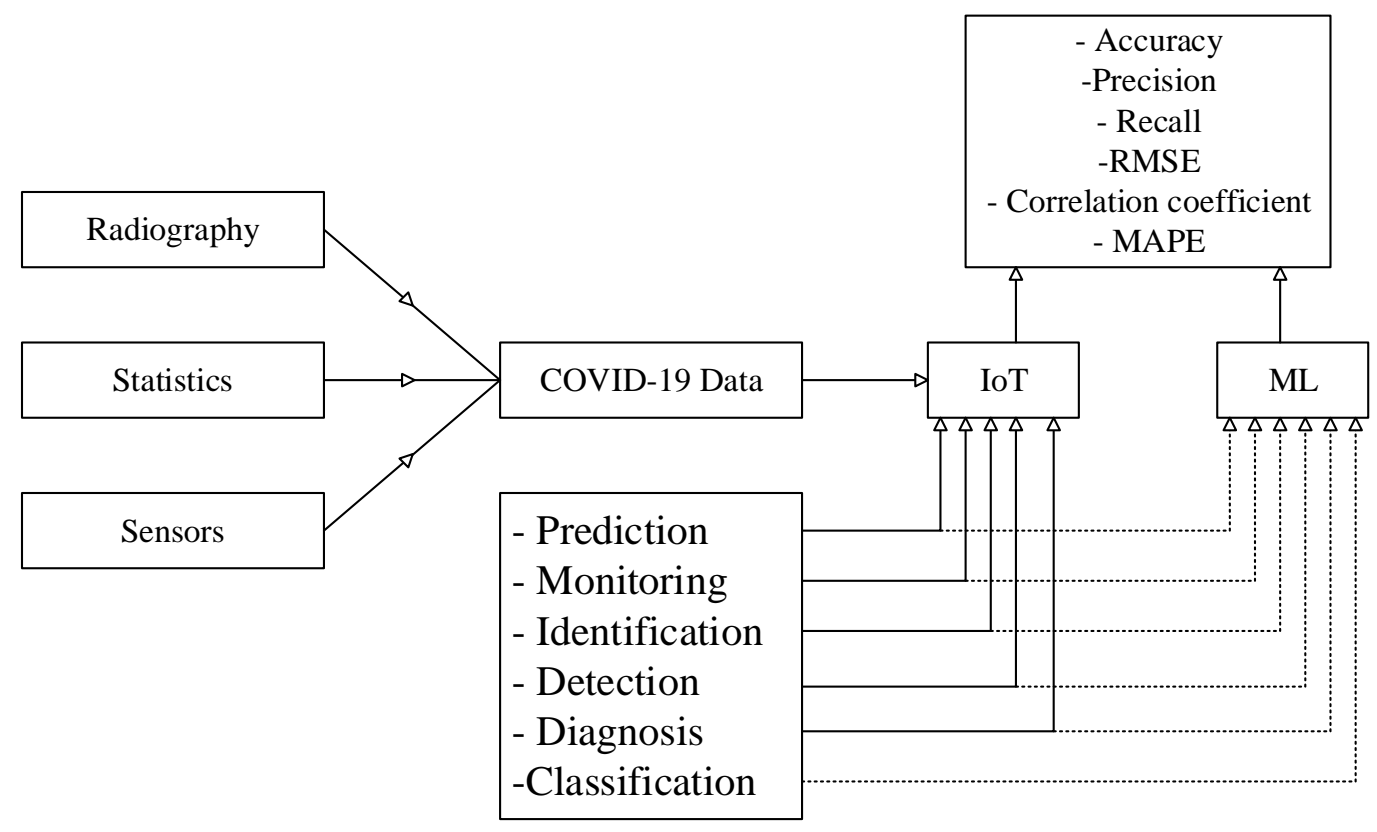

Figure 3. Main contributions of the current study

There is a need to categorize the main applications of IoT and the relevance technique in accordance with the COVID-19. Table 3 presents the main contributions of the study for the application of IoT and integrated IoT-ML based techniques.

Table 3. The main contribution of the study for the application of IoT based techniques

\begin{tabular}{|c|c|c|c|c|c|}
\hline Methodology & Prediction & Monitoring & Detection & Identification & Diagnostic \\
\hline IoT & 口 & 田 & У & & \\
\hline IoT-DNN & & & & & 口 \\
\hline IoT-RCNN & & & & & 口 \\
\hline IoT-SVM & & 口 & & 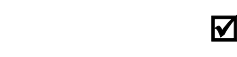 & \\
\hline IoT-ANN & & 口 & & $\nabla$ & \\
\hline IoT-Naïve Bayes & & 口 & & $\square$ & \\
\hline
\end{tabular}




\begin{tabular}{lccc}
\hline IoT-K-NN & $\square$ & & $\square$ \\
IoT-DT & $\square$ & & \\
IoT-Fog based & $\square$ & & \\
IoT-Deep edge computing & $\square$ & & \\
Wireless sensors & $\square$ & & $\square$ \\
IoT based SEIR & & $\square$ & \\
IoT-IT & $\square$ & $\square$ & \\
\hline
\end{tabular}

As shown in Table 3, IoT based technology requires using ML-based techniques for completing the task. Figure 4 presents the share of each methodology in the applications by percentage.

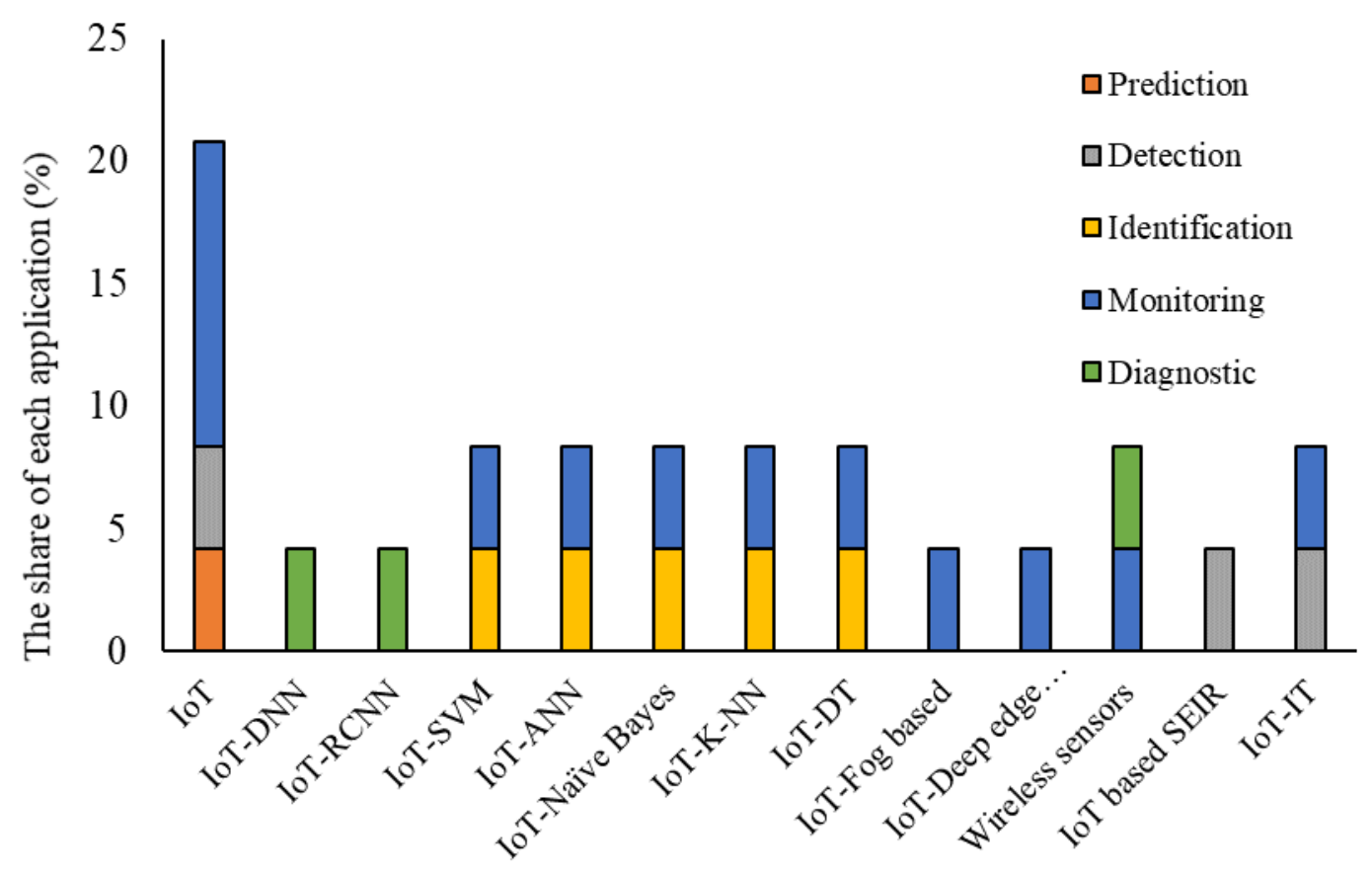

Figure 4. The share of each application $(\%)$

As shown in Figure 4, IoT has been used more than other applications to monitor and detect COVID19 cases. While, it has been less popular in the identification.

\subsection{ML techniques for pandemic prediction of COVID-19}

Utilizing ML platform led to reducing the adverse effects of the disease and accelerating the healing process [51]. Combination of AI and ML have led to advances in treatment, medication, screening, prognosis, contact tracking and the drug/vaccine development process and reduced human intervention 
in medical performance [71]. ML is also used as a tool for management of virtual queues to prevent crowds in physical waiting rooms or long queues. It is used to predict waiting times and implement calls in a privacy manner in conjunction with the cell-phone platform [72].

ML method is widely used in data analysis by producing an analytical model intelligently. This method is a subset of artificial intelligence that analyzes data and produces a model for estimating, categorizing, optimizing, predicting, identifying problems, as well as decision making [73, 74].

New computing technologies have made the problems assessed by ML-based techniques today a little different from the way they are analyzed based on past technologies [75]. These techniques began to evolve from pattern recognition to a comprehensive theory of the ability of computers to perform specific tasks without the need for special planning [76, 77].

In the field of medicine and treatment, ML is known as one of the most practical tools for analyzing medical data, identifying, predicting, and even treating in different situations. With the advancement of medical science in today's world and the production of large volumes of medical data, there is an urgent need to analyze this data [78]. Figure 5 presents the main applications of ML-based techniques for medical science to tackle COVID-19 pandemic. Identifying the prevalence, effective parameters in the eradication of the virus, identifying patients in the early stages, patients' pattern behaviors, and predicting outbreak and mortality rates can be considered practical and effective areas of ML-based techniques $[1,79]$.

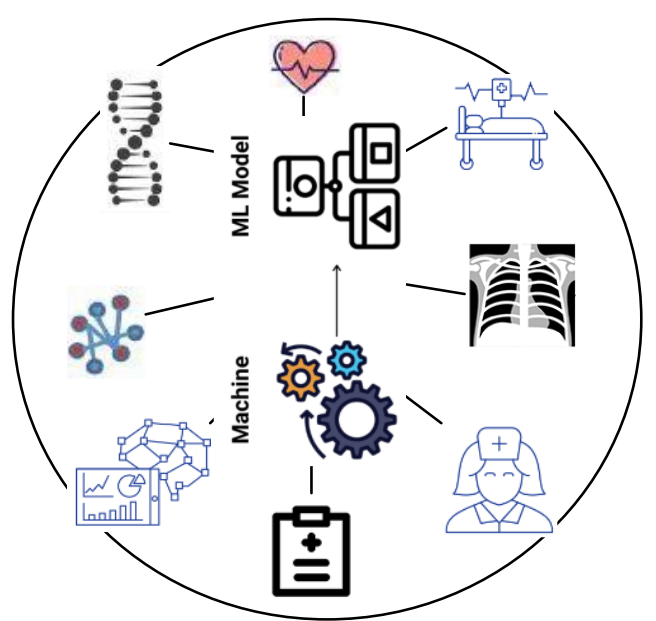

Figure 5. the main applications of ML-based techniques for medical science

Table 4 presents the highlighted studies for the application of ML-based techniques for handling COVID-19. Similar to Table 3, Table 4 discuss them in four columns. Objective column that briefly describes the main objective of each study. Methodology/proposed algorithm presents the main algorithm and procedure employed by each study. Keyword indicates the main points and concentration of the study and finally, application section presents the field of the application of each method.

Table 4. ML-based techniques for COVID-19 


\begin{tabular}{|c|c|c|c|c|c|}
\hline Order & aim & method & Key words & application & Ref \\
\hline 1 & $\begin{array}{l}\text { To develop a mask face } \\
\text { detection model }\end{array}$ & $\begin{array}{l}\text { deep transferring } \\
\text { learning (ResNet50) as } \\
\text { classifier and SVM to } \\
\text { be compared with } \\
\text { ensemble method }\end{array}$ & $\begin{array}{l}\text { deep transferring learning, } \\
\text { SVM, and ensemble }\end{array}$ & Detection & [80] \\
\hline 2 & $\begin{array}{l}\text { To employ ML based } \\
\text { platform as a healthcare } \\
\text { application to proper } \\
\text { decision making for } \\
\text { COVID-19 detection }\end{array}$ & $\begin{array}{l}\text { Integration of random } \\
\text { forest, Gaussian nave } \\
\text { bias and Generative } \\
\text { adversarial network }\end{array}$ & $\begin{array}{l}\text { Artificial intelligence, } \\
\text { Cloud/fog computing, IoT }\end{array}$ & Detection & [81] \\
\hline 3 & $\begin{array}{l}\text { To propose an AI based } \\
\text { technique integrated by CT } \\
\text { scan and chest X-ray images } \\
\text { to identify, and predict the } \\
\text { positive infected patients }\end{array}$ & pre-trained CNN & $\begin{array}{l}\text { COVID-19, DT, X-ray } \\
\text { images, AI }\end{array}$ & $\begin{array}{l}\text { Identification } \\
\text { and diagnosis }\end{array}$ & [82] \\
\hline 4 & $\begin{array}{l}\text { To employ a novel CNN } \\
\text { architecture for classifying } \\
\text { COVID-19 from chest X- } \\
\text { rays. }\end{array}$ & $\mathrm{CNN}$ architecture & $\begin{array}{l}\mathrm{DL}, \mathrm{CNN} \text {, mine data } \\
\text { patterns }\end{array}$ & $\begin{array}{l}\text { Classify and } \\
\text { identification }\end{array}$ & [83] \\
\hline 5 & $\begin{array}{l}\text { To develop an AI based } \\
\text { methods for fast diagnosis } \\
\text { of COVID-19 cases }\end{array}$ & $\begin{array}{l}\text { ResNet-101 in } \\
\text { comparison with } \\
\text { Radiology data }\end{array}$ & AI, CNN, ResNet-101 & Diagnosis & [84] \\
\hline 6 & $\begin{array}{l}\text { To detect COVID-19 } \\
\text { promptly using CNN }\end{array}$ & CNN technique & DL, CNN, Squeeze Net & Detection & [85] \\
\hline 7 & $\begin{array}{l}\text { To develop and test a new } \\
\text { computer-aided diagnosis } \\
\text { (CAD) to investigate } \\
\text { COVID-19 }\end{array}$ & $\mathrm{CNN}$ & CNN, DL, CAD & Diagnosis & [36] \\
\hline 8 & $\begin{array}{l}\text { To propose an intelligence } \\
\text { computer-aided model to } \\
\text { support daily clinical } \\
\text { applications }\end{array}$ & $\begin{array}{l}\text { convolution neural } \\
\text { network (CNN) with } \\
\text { SVM classifier } \\
\text { architecture on chest } \\
\text { X-ray }\end{array}$ & $\begin{array}{l}\text { Medical decision support } \\
\text { system; } \\
\text { Deep learning }\end{array}$ & Detection & [86] \\
\hline 9 & $\begin{array}{l}\text { To develop an AI based } \\
\text { model for proper screening } \\
\text { and monitoring COVID-19 }\end{array}$ & AD3D-MIL & screening, CAD, DL, ML & Monitoring & [87] \\
\hline 10 & $\begin{array}{l}\text { To present a CNN based } \\
\text { technique for early COVID- } \\
19 \text { diagnosis from chest X- } \\
\text { ray }\end{array}$ & $\mathrm{CNN}$ & AI, CNN, DL & Diagnosis & [35] \\
\hline 11 & $\begin{array}{l}\text { To investigate a medical } \\
\text { decision support system by } \\
\text { CNN }\end{array}$ & $\mathrm{CNN}$ & $\begin{array}{l}\text { Decision support; CNN; } \\
\text { DL; ML }\end{array}$ & Diagnosis & [88] \\
\hline
\end{tabular}


12 To propose an intelligent methodology to diagnosis the COVID-19 cases

13 To study the utility of AI in a prompt and accurate diagnosis of COVID-19 in the presence of chest X-ray images

14 ML-based classification approach for handling COVID-19

15 ML-based classification algorithm for handling infectious diseases, such as COVID-19

16 To detect the COVID-19 cases using RNN technique

17 To present a fuzzy rule basing system to predict COVID-19 daily cases

18 To present a multi-scale discriminative segmentation network to detect COVID19

19 To develop a hybrid AI technique for the prediction of COVID-19

20 To present a solution for identifying pneumonia using CXR images

21 to examine the emotions expressed by people using social media to track and diagnosis sentiment behind COVID-19

22 To propose ML-based approach for the forecasting of COVID-19 cases
The multi-criteria decision-making

(MCDM) using

TOPSIS in the presence of SVM based classifier

pre-trained $\mathrm{CNN}$

Extreme gradient boosting (XGBoost) model

KNN, SVM, DT and LR

LSTM architecture of RNN method for detection based on Cough sound, Breathing sound and voices

Fuzzy rule based

MSD-Net

Integrated natural language processing module and the LSTM

GCNN

LR, Multinomial

Naïve Bayes, DT, RF, SVM and XGBoost classifiers

MLP and ANFIS
COVID-19, CT, DL

COVID19 diagnostic, machine learning, benchmarking; TOPSIS,

\author{
AI; COVID-19; machine \\ learning, Convolutional \\ Neural Networks
}

dinucleotide frequencies, feature representations, genomic signatures, human pathogens, ML, extreme gradient boosting

machine learning,

\section{AI, DL, RNN}

COVID-19, AI, fuzzy rule based inference

COVID-19, prediction, epidemic model, hybrid AI, detection

GCNN, Computed Tomography, Chest X-Ray, AI

twitter; emotions; sentiment Detection analysis; pandemic; domain-specific; COVID19; ML; dataset

ML, COVID-19 cases, prediction, detection diagnosis

Classification

Detection Prediction,

Classification

Detection

\author{
Detection
}



COVID19 cases

According to Table 4, ML-based techniques are employed for detection, identification, monitoring, diagnosis, prediction and classification purposes in the presence of the COVID-19 dataset. Figure 6 presents the summary of each application, separately. Singh and Kaur employed ML based platform using hybrid random forest, Gaussian Naïve Bayes and Generative adversarial network as a healthcare application to detect COVID-19 cases [81]. Vinod et al developed a pre-trained CNN method as an ML based technique integrated by CT scan and chest X-ray images to identify, detect, and predict the positive infected patients [82]. Ardakani et al developed an ML based technique (ResNet) for fast diagnosis of COVID-19 cases compared to radiology data [84]. Polsinelli et al developed a study to detect COVID-19 promptly using CNN as a frequently used DL-based architecture [85]. Nour et al proposed an intelligence computer-aided model based on CNN with SVM classifier architecture on chest X-ray to support daily clinical applications [86]. Chowdhury et al investigated the utility of AI in the rapid and accurate detection of COVID-19 in the presence of chest X-ray images [90]. Sethi et al employed Logistic Regression (LR), Multinomial Naïve Bayes, Decision Tree (DT), Random Forest (RF), SVM and XGBoost classifiers analyze the emotions expressed by people using social media to monitor and detect sentiment behind COVID-19 [98]. Ardabili et al developed ML-based techniques for the prediction of COVID-19 outbreaks [1]. In another study, Ardabili et al also employed hybrid ML-based technique (Multilayered perceptron integrated by grey wolf optimizer) for the globally prediction of COVID-19 cases [79]. In addition, Loey et al employed DL based ResNet method in the presence of SVM-based classifier was employed to detect a mask face [80].

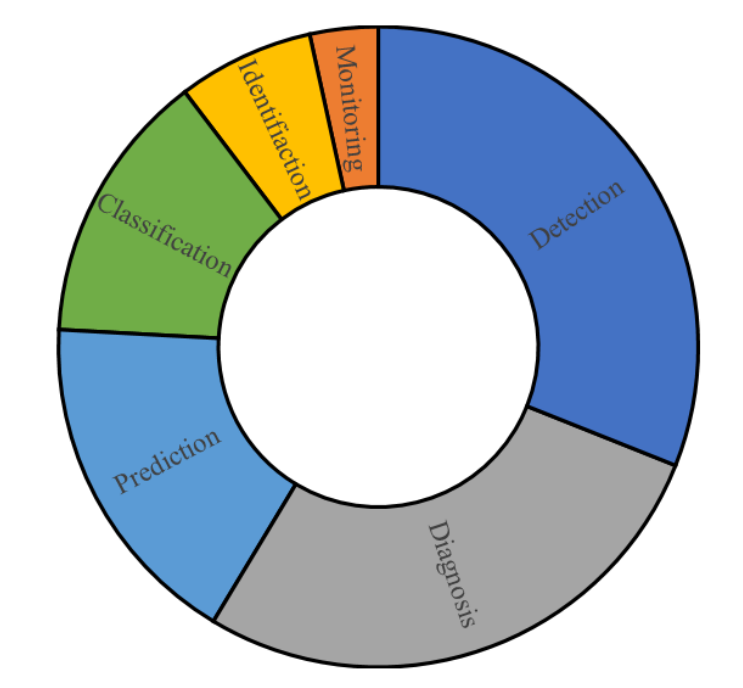

Figure 6. The share of each application type for ML-based systems 
According to Figure 6, detection, diagnosis, and prediction can be considered as the main categories of the application of ML-based methods in COVID-19. In general, one of the main sections of analyzing IoT-based and ML-Based techniques applied for a specific field is their evaluation in terms of accuracy, error ${ }_{2}$ or in other word performance of the model. Table 5 presents the evaluation criteria employed for each model.

Table 5. The main evaluation criteria for analyzing the performance of models

\begin{tabular}{|c|c|c|c|c|c|c|c|c|c|c|c|}
\hline & 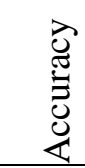 & 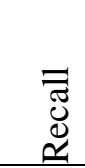 & 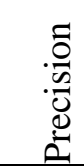 & 己ֶ, & 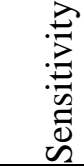 & 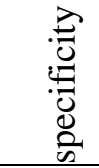 & 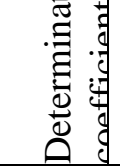 & $\sum_{\underline{\underline{N}}}^{\underline{\underline{N}}}$ & $\frac{1}{2}$ & $\sum^{\frac{1}{1}}$ & $\begin{array}{l}0 \\
0 \\
0 \\
0 \\
\frac{1}{1} \\
\end{array}$ \\
\hline $\begin{array}{l}\text { deep transferring learning } \\
\text { (ResNet50) }\end{array}$ & $\nabla$ & & & & & & & & & & \\
\hline RF-NB-GAN & & $\square$ & 口 & & & & & & & & \\
\hline $\mathrm{CNN}$ & 口 & $\nabla$ & 口 & 口 & & & & & $\nabla$ & & $\nabla$ \\
\hline ResNet-101 & 口 & & & & 口 & 口 & & & & & \\
\hline AD3D-MIL & 口 & & & 口 & & & & & & & \\
\hline TOPSIS & & & & & & & 四 & & & & \\
\hline XGBoost & 口 & & & & & & & & & & \\
\hline $\mathrm{kNN}$ & 口 & & & & & & & & & & \\
\hline SVM & 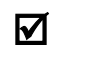 & & & & & & & & & & \\
\hline DT & У & & & & & & & & & & \\
\hline LR & 口 & & & & & & & & & & \\
\hline LSTM & 口 & & & 口 & & & & & & & 口 \\
\hline Fuzzy & & & & & & & 口 & 口 & & 口 & \\
\hline
\end{tabular}




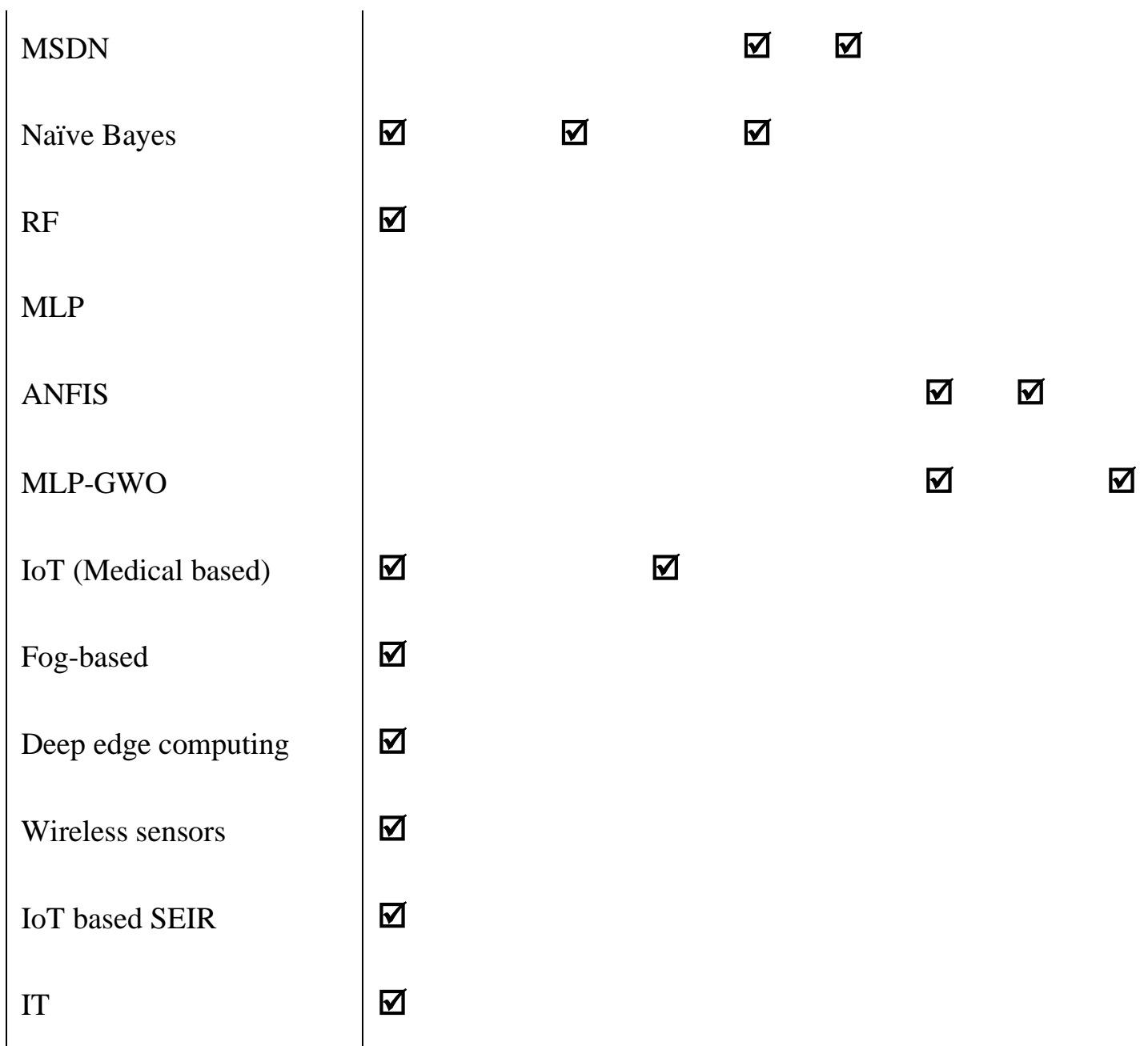

According to Table 5, accuracy, followed by recall and precision parameters have owned the highest portion of the evaluation criteria employment for analyzing COVID-19 based dataset using IoT and ML-based techniques. In the following, Table 6 is generated from Table 4 for indicating the share of each ML-based techniques for each application and their main contributions. According to Table 6, ResNet as an architecture of deep learning methods followed by CNN, XGBoost, SVM, DT and LR have been used more often to tackle work with COVID-19 related data.

Table 6. The main contribution of ML-based techniques in COVID-19 applications

\begin{tabular}{lrrrrrr}
\hline & Prediction & Monitoring & Diagnosis & Identification & Detection & Classification \\
\hline ResNet50 & $\square$ & $\square$ & $\square$ & $\square$ & $\square$ & $\square$ \\
RF-Naïve bayes-GDN & & & & & $\square$ & \\
CNN & & & & & $\square$ & \\
ResNet-101 & & & $\square$ & $\square$ & $\square$ \\
AD3D-MIL & & & $\square$ & &
\end{tabular}


TOPSIS

XGBoost

kNN

SVM

DT

LR

LSTM

Fuzzy

multi-scale discriminative network

GCNN

Naïve Bayes

$\mathrm{RF}$

MLP

ANFIS
曰

曰

च
可可

च

田可

可 可

可 可

甲

田

口

च

MLP-GWO

可

口

四

Figure 7 presents the share of different ML methods for different tasks to tackle COVID-19 pandemic. As it is clearly indicated in this figure, ResNet followed by CNN are the most common application of ML in this field. This can be due to the model's nature for handling different applications like monitoring, detection, identification, classification, and diagnosis. While, other methods are capable to do limited number of applications.

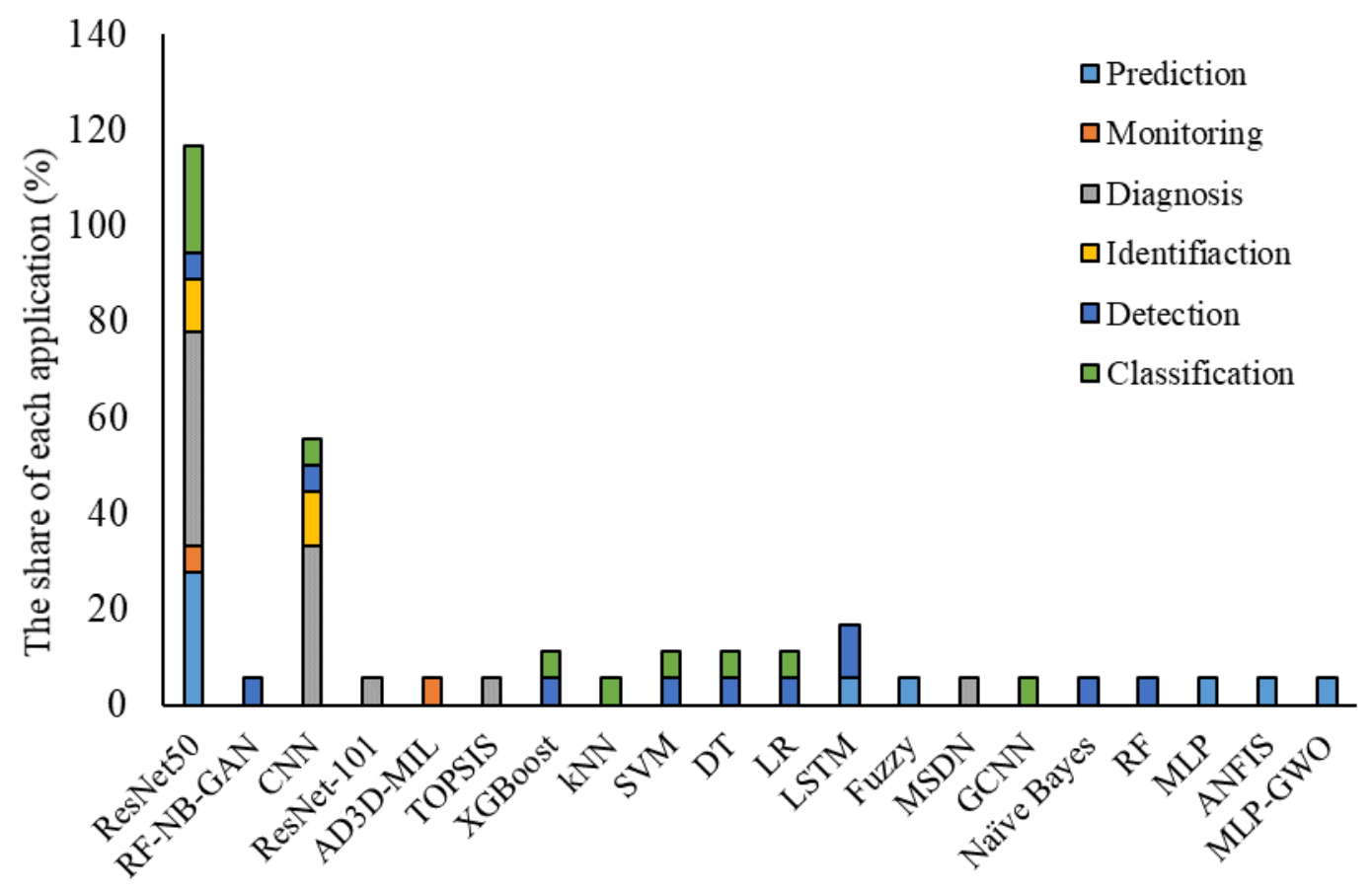


Figure 7. The share of each application $(\%)$

\subsection{Evaluation criteria}

Models developed using ML and IoT-ML requires an evaluation step for recognizing their performance and accuracy values. According to the studies reviewed, the most effective and frequently used evaluation criteria are including Accuracy, Recall, Precision, Root mean square error (RMSE), Correlation coefficient and Mean absolute percentage error (MAPE). These criterias compare the models' output and actual values and provides a comparison score [1,79]. In the present study, we employed the criteria values reported by each study for evaluating and comparing the models. Table 7 presents the main criteria for evaluation.

Table 7. The main evaluation metrics

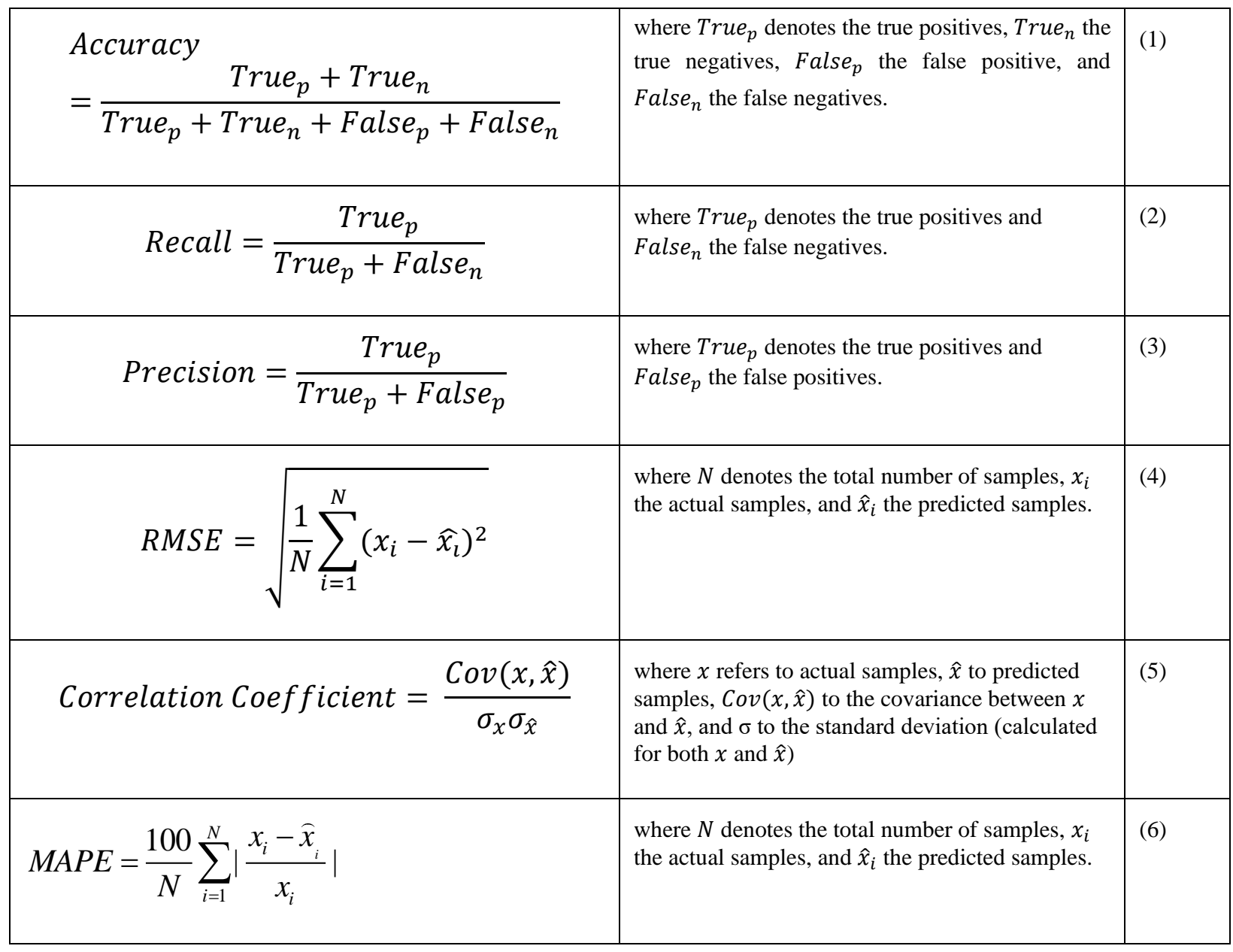

\section{$3 \quad$ Main findings and evaluations}


This section presents the main findings of IoT based techniques (Table 8) and ML-based techniques (Table 9). Each table includes two main columns called findings and pros. and cons.

Table 8. The main findings of the study for the application of IoT-based techniques

\begin{tabular}{|c|c|c|c|}
\hline Order & Findings & Pros. and Cons. & Ref. \\
\hline 1 & $\begin{array}{l}\text { The proposed solution can identify and track the } \\
\text { infected individual and successfully tracks all people } \\
\text { who are in the area of disease spread }\end{array}$ & $\begin{array}{l}\text { This framework integrates symptom information as a rapid and } \\
\text { efficient approach, thus tracking the prevalence of the disease }\end{array}$ & {$[55]$} \\
\hline 2 & DL applications are vulnerable to coronavirus attacks & The method is very vulnerable and requires further studies & [56] \\
\hline 3 & The model provides an accuracy of $98 \%$ for detection & $\begin{array}{l}\text { Combining DL and the IoT makes it easier for radiologists to control } \\
\text { the spread of the virus }\end{array}$ & [57] \\
\hline 4 & $\begin{array}{l}\text { According to results, all the techniques, except the } \\
\text { Decision Stump, OneR, and ZeroR provided accuracies } \\
\text { values more than } 90 \%\end{array}$ & $\begin{array}{l}\text { The proposed platform reduced the communicable diseases using early } \\
\text { detection of cases and provided tracking the recovered cases, and a } \\
\text { better understanding of the infections }\end{array}$ & {$[58]$} \\
\hline 5 & $\begin{array}{l}\text { IoT reduces clinical cost and optimizes treatment } \\
\text { outcome of the patients }\end{array}$ & $\begin{array}{l}\text { The platform improves patient satisfaction and decreases readmission } \\
\text { rate in the hospital }\end{array}$ & [59] \\
\hline 6 & $\begin{array}{l}\text { The system can assist tracking the daily activities and } \\
\text { decrease the risk of exposure to the COVID-19 }\end{array}$ & $\begin{array}{l}\text { The app announces the user to keep a physical distance of } 2 \mathrm{~m} \text {. Also, a } \\
\text { Fuzzy-based technique evaluates the environmental risk and user health } \\
\text { to estimate the risk of real time spreading. This platform can } \\
\text { successfully reduce the coronavirus spread }\end{array}$ & {$[60]$} \\
\hline 7 & The platform detects and tracks the infected person & $\begin{array}{l}\text { The platform tracks COVID-19 and improves infected person and } \\
\text { keeps the dataset for further analysis }\end{array}$ & [61] \\
\hline 8 & $\begin{array}{l}\text { The provided package enhances the testing process for } \\
\text { increasing the efficiency of the system }\end{array}$ & $\begin{array}{l}\text { This approach will increase the maximum collaboration from the } \\
\text { employees }\end{array}$ & {$[62]$} \\
\hline 9 & $\begin{array}{l}\text { This platform is a cost-effective, safety-critical mobile } \\
\text { robotic technology and successfully copes with } \\
\text { diagnosis task Also the multiple diagnostic devices } \\
\text { increases the detection accuracies }\end{array}$ & $\begin{array}{l}\text { The system effectively provides a complete diagnosis and figuring out } \\
\text { COVID-19 patients also contains multiple diagnostic devices, without } \\
\text { any need for human interferences }\end{array}$ & [63] \\
\hline 10 & $\begin{array}{l}\text { The robot technology protect virus affected persons. } \\
\text { The system is also recognizing the patient's Gesture and } \\
\text { tracking the instructions }\end{array}$ & $\begin{array}{l}\text { The robot collects data from patient performs tasks without image } \\
\text { processing system }\end{array}$ & [64] \\
\hline 11 & IoT-based technology prevent the global pandemic & $\begin{array}{l}\text { improves the control and tracking of a fast-spreading virus such as } \\
\text { coronavirus }\end{array}$ & {$[65]$} \\
\hline 12 & $\begin{array}{l}\text { The proposed methodology is sustainable for disease } \\
\text { tracking by an early identification of cases }\end{array}$ & $\begin{array}{l}\text { This technique can successfully handles both governments and other } \\
\text { decision-making authorities }\end{array}$ & [66] \\
\hline 13 & This system improves the decision-making procedure & $\begin{array}{l}\text { The system is connected through cloud computing and effectively } \\
\text { supports the real-time data }\end{array}$ & [67] \\
\hline 14 & $\begin{array}{l}\text { Edge computing improved the findings on the } \\
\text { decentralized load of face recognition }\end{array}$ & The platform enhances the robustness of detection and diagnosis & [68] \\
\hline 15 & $\begin{array}{l}\text { The proposed system could successfully cope with the } \\
\text { task }\end{array}$ & $\begin{array}{l}\text { IoT equipped ML can successfully save, and visualize monitoring the } \\
\text { volunteers }\end{array}$ & [69] \\
\hline 16 & $\begin{array}{l}\text { This study suggests that integrated and hybrid } \\
\text { techniques will follow up the near future, using } \\
\text { simulation, and forecasting purposes }\end{array}$ & A higher degree of safety and privacy for humanity & [34] \\
\hline 17 & $\begin{array}{l}\text { The platform employed for the study have an effective } \\
\text { role in the success of pandemic handling }\end{array}$ & The platform increases accessibility to the proper dataset & [70] \\
\hline
\end{tabular}

According to Table 8, most of these studies lack numerical analysis for the method performance. One of the main reasons can be the nature of IoT technique which goes through a practical process and shows its performance in practical applications and does not need to provide numerical statistics. In all these applications, IoT could successfully cope with the task. Such that, IoT provided a fast and 
efficient approach of tracking the disease spread [55]. On the other hand, it can be employed as a realtime framework to minimize the impact of communicable diseases through early detection of cases [56]. In the study by Singh et al, IoT technology successfully increased patient satisfaction and reduces readmission rate in the hospital [59]. However, for detection purposes, there is a need for integrating IoT platform with ML-based techniques. In the study by Rahman et al, DL applications with IoT platform provided promising findings to detect $\mathrm{AE}$ attacks. However, there is a need for further research, attention, and implementation of appropriate defense mechanisms, safeguards, and controls [56]. Kolhar et al employed Multi-task Cascaded Convolutional Network architecture (MCCNN) and findings claimed that the efficiently integrated by Raspberry Pi increased the robustness of detection and recognition [68].

Table 9. The main findings of the study for the application of ML-based techniques

\begin{tabular}{lll}
\hline Order & \multicolumn{1}{c}{ Results } & \multicolumn{1}{c}{ Pros. and cons. } \\
\hline 1 & $\begin{array}{l}\text { The SVM classifier in the presence of RMFD, SMFD } \\
\text { and LFW dataset achieved } 99.64,99.49 \text { and } 100 \% \\
\text { testing accuracy values. }\end{array}$ & $\begin{array}{l}\text { The proposed model provided lowest processing time and } \\
\text { highest accuracy }\end{array}$ \\
$\begin{array}{l}\text { Recall=0.93, Precision=0.871 with lower processing } \\
\text { time }\end{array}$ & $\begin{array}{l}\text { The system is cost-effective by reducing processing time } \\
\text { and sustainable by increasing the accuracy values } \\
\text { considerably. The proposed framework can also be used to } \\
\text { prioritize patients who require an ambulance. }\end{array}$
\end{tabular}

Ref

[80] and LFW dataset achieved 99.64, 99.49 and 100\% Recall=0.93, Precision=0.871 with lower processing and sustainable by increasing the accuracy values considerably. The proposed framework can also be used to

3 Accuracy $=93 \%$ and recall score $=88 \%$ using chest $x-$ ray images

The proposed method can successfully help radiologist's prompt detection of coronavirus cases

a channel-shuffled dual-branched $\mathrm{CNN}$ architecture can effectively learn salient features and increases the accuracy and precision values of the modeling

$5 \quad$ Sensitivity $=100 \%$, specificity $=99.02 \%$ and accuracy $=99.51 \%$ and for radiology data, sensitivity $89.21 \%$, specificity $=83.33 \%$ and accuracy $=86.27 \%$

This model is low cost and is used as a complementary method during CT imaging

$6 \quad$ Accuracy $=85.03 \%$, sensitivity $=87.55 \%$, specificity $=81.95 \%$, precision $=85.01 \%$ and $\mathrm{F} 1$ core $=86.20 \%$

Higher classification rate by analyzing thousands of images

$7 \quad$ Accuracy $=94.5 \%$, confidence interval $=95 \%$, sensitivity $=98.4 \%$ and specificity $=98.0 \%$

$8 \quad$ Accuracy $=98.97 \%$, sensitivity $=89.39 \%$, specificity $=99.75 \%$, and an F-score $=96.72 \%$

develops a DL-based CAD scheme of chest X-ray images and improves detecting COVID-19 infected

reduces the misdiagnosis rates, and improves evaluation rates and detects positive COVID-19 infections

Reliable screening of COVID-19 from chest CT score $=95.7 \%$.

$1096 \%$ of accuracy

The proposed model performance is clinically validated with expert radiologists

11 Accuracy of $99.62 \%$ and $96.70 \%$. Average recall value of $99.63 \%$ and $96.69 \%$ respectively for binary and multiclass

12 Correlation coefficient $=0.9899$

Automated medical diagnostics for enhancing decision making rates

providing significant variance for each criterion

13 Accuracy $=99.7 \%$, precision $=99.7 \%$, and Improving the speed and accuracy of COVID-19 detection 
$14 \quad 86 \%$ accuracy for the task of classifying

15

16

17

21 Accuracy for both SVM and

Decision Tree could provide the maximum value by average value of $93 \%$

22 RMSE and CC values for five countries including, China, Italy, USA, Iran and Germany

23

Accuracy of $88,91,87$ and $89 \%$ for kNN, SVM, DT and LR, respectively

F1-score of 97.9, 98.8 and 92.5\%, AUC of 97.4, 98.8 and $84.4 \%$ and accuracy of $97,98.2$ and $88.2 \%$ respectively for Cough sound, Breathing sound and voices, respectively.

$$
\mathrm{R} 2=0.96, \mathrm{RMSE}=254, \mathrm{MAE}=186
$$

Sensitivity and specificity of 0.8645 , and 0.9889 .

MAPEs $=0.52 \%, 0.38 \%, 0.05 \%$, and $0.86 \%$ respectively for the next six days in Wuhan, Beijing, Shanghai, and countrywide

Accuracy $=98.84 \%$, Precision $=93 \%$,

Sensitivity $=100 \%$, and Specificity $=97.0 \%$

MAPE $=13.15 \%$ and $\mathrm{CC}=0.99$
The proposed model could successfully improve the classification accuracy

The proposed method can be applied anywhere, without prior training or calibration

To improve the COVID-19 detection through a costeffective approach

The proposed method could successfully estimate the number of daily cases

This model provides automated and accurate segmentation of CT images

To minimize the errors of the prediction and to enhance the detection efficiency

The proposed model improved classification rate in comparison with ReseNet18, ReseNet50, Squeeze net, DenseNet-121, and Visual Geometry Group

Higher accuracy for perceiving the perception of people infected by COVID-19

The proposed models enhanced the forecasting rate of COVID-19 cases

The proposed models increased the forecasting rate of COVID-19 cases

According to findings of Table 9, the most share of studies developed by ML-based techniques for handling COVID-19 based dataset provided performance criteria. The most share of the performance criteria, according to Figure 8 is related to accuracy factor. Accuracy factor is a general and normalized factor. Therefore, it can be employed for comparing the ML-based methods with different datasets. Figure 9 presents the accuracy values for each model for comparing their performance in handling COVID-19 dataset. 


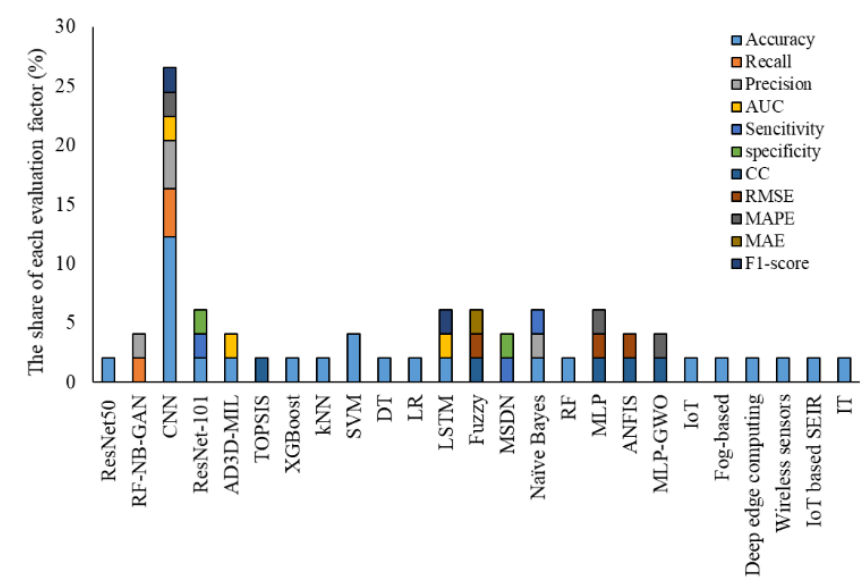

Figure 8. The share of each evaluation factor (\%) for analyzing results

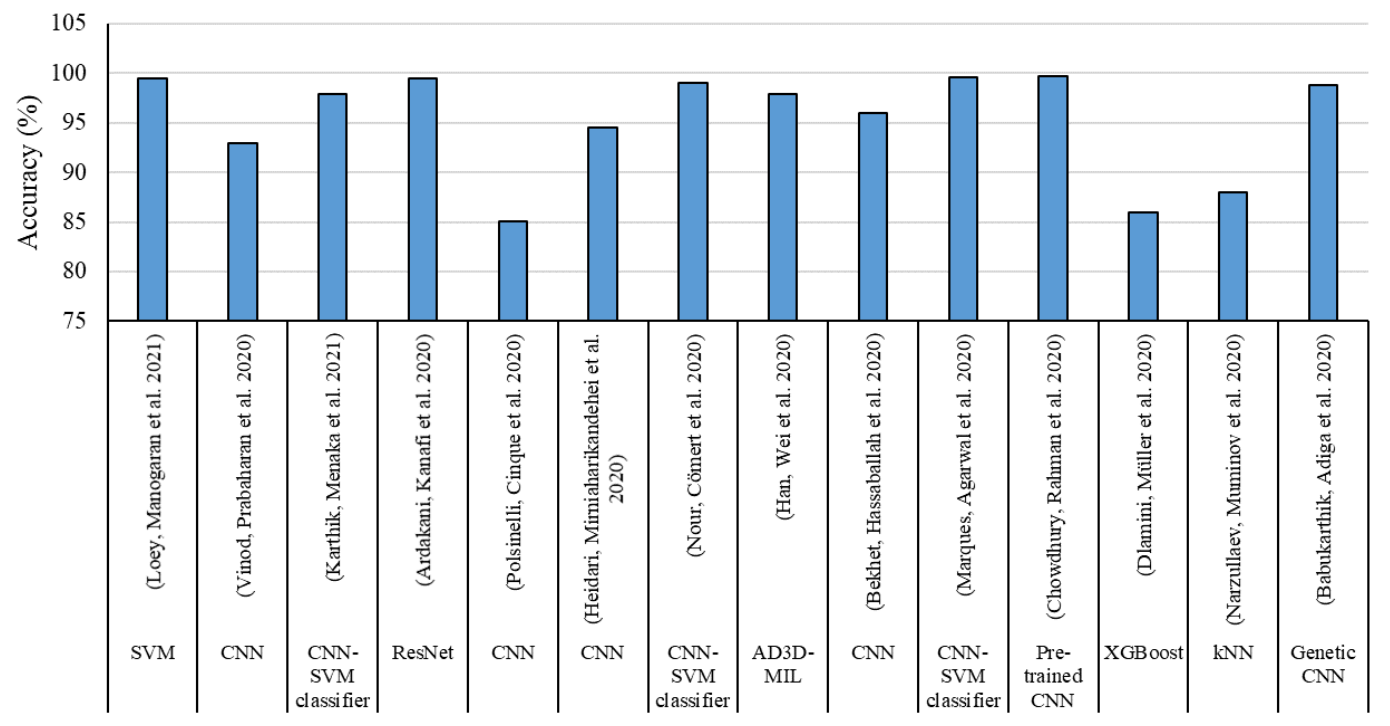

Figure 9. Accuracy values

Figure 9 indicates CNN with SVM classifier, Genetic CNN and pre-trained CNN followed by ResNet, provided highest accuracy values. On the other hand, the lowest accuracy was related to single CNN followed by XGboost and KNN techniques.

\subsection{Challenges and limitations}

Nowadays, when the world is struggling with COVID-19 disease, every innovation and technology is used to fight this disease. Like many other areas, healthcare requires the support of new technologies 
such as IoT, and ML. Exploring of disease-related dataset, data preparation, prevention, and control of infectious diseases, has become one of the main purposes of AI. IoT and ML have a vital personality in better understanding, dealing with the COVID-19 crisis, and discovering the COVID-19 vaccine. ML-based technology allows computers to predict the pattern and speed of disease transmission with the intelligence they have and by mimicking large amounts of data. This result-oriented technology is employed for proper screening, analysis, forecasting and tracking of current and potential future patients. AI uses information from people with coronary heart disease, improved and dead people as tracking data.

To combat the spread of the corona virus, IoT-based methods of communicating with patients provide transparency and a better understanding of how the virus is spread, and strengthen the treatment and research process. ML is one of the new technologies in tracking the spread of the virus and finding effective parameters in it. The ML method can successfully identify high-risk patients and predict the necessary measures to deal with possible infections to reduce the point of effect of the disease. MLbased methods can estimate the risk of patient mortality through previous analysis. This technique improves the planning, treatment, and reduction of patients and is a complementary medical tool that works with data and evidence. On the other hand, this technology improves decision making and reduces the cost of treatment and diagnosis. At the same time, in the field of medical imaging, ML tools helps to recognize the patterns in the images and strengthen the ability of radiologists to diagnose the possibility of disease and early diagnosis of the disease.

One of the main limitations of IoT, and ML-based techniques for applications in COVID-19 is the lack of a complete dataset. This can be due to the unique development of models by limited data for a specific application within the same data field. The purpose of using IoT, AI, or ML-based techniques is to solve a specific problem in the real world with a real application that requires the use of special hardware and equipment. There are limitations in the cost and availability of developing and equipping communication hardware in therapeutic, diagnostic, estimation, and forecasting applications for IoT technology or ML-based techniques. Also, there are limited best practices available for IoT developers. The lack of IoT edge authentication and licensing standards has led to restrictions on the application and enactment of laws, regulations, and policies in the use of this technology, and this has led to the absence of IoT-based incident response activities as the best methods. All of these limitations mean that there is still no focus on identifying ways to gain situational awareness of the security of IoT assets in a medical complex.

\section{Conclusion}

The present study categorizes the applications of IoT, IoT-ML, and ML-based techniques to tackle COVID-19-related problems. The main applications are categorized into monitoring, detection, identification, classification and diagnosis. Studying, comparing and investigating of these applications requires a proper judgment about the performance and effectivity of outputs. According to a deep consideration about the evaluation criteria, it has been investigated that the accuracy, followed by recall and precision parameters have owned the highest portion of the evaluation criteria employment for analyzing COVID-19 based dataset using IoT and ML-based techniques.

Most of the studies lack of numerical analysis for the method performance. One of the main reasons that can be the nature of IoT technique which goes through a practical process and shows its performance in practical applications. In all the applications, IoT could successfully cope with the 
tasks. Such that, IoT provided a fast and efficient approach of tracking the disease spread. Most of the studies developed by ML-based techniques for handling COVID-19 based dataset provided performance criteria. The most popular performance criteria is related to accuracy factor. It can be employed for comparing the ML-based methods with different datasets. According to the results, CNN with SVM classifier, Genetic CNN and pre-trained CNN followed by ResNet, provided highest accuracy values.

Policy-making in the field of COVID-19 disease to examine the weaknesses and strengths and vulnerabilities of society in terms of the penetration of pathogenic viruses can be considered as additional measures and future studies. On the other hand, the study of collective behaviors can also be considered as perspectives to complete studies in order to prevent similar social harms and reduce costs incurred and not to surprise human life.

\section{$5 \quad$ Conflict of Interest}

The authors declare that the research was conducted in the absence of any commercial or financial relationships that could be construed as a potential conflict of interest.

\section{$6 \quad$ Author Contributions}

SSB and SA designed the study; SA, AY, BP, and AKK, AM wrote the paper. SS, AB, HAR, and AD edited the manuscript. SA, AY, BP carried out all the analyses. SSB, SA, AY, BP generated all figures and all tables. HAR and AB were not involved in any analyses. All authors have read and approved the final version of the paper.

1. Ardabili, S.F., et al., Covid-19 outbreak prediction with machine learning. 2020. 13(10): p. 249.

2. Ciotti, M., et al., The COVID-19 pandemic. 2020. 57(6): p. 365-388.

3. Mahmoudi, M.R., et al., Principal component analysis to study the relations between the spread rates of COVID-19 in high risks countries. Alexandria Engineering Journal, 2021. 60(1): p. 457-464.

4. $\quad$ Kumar, A., et al., A review of modern technologies for tackling COVID-19 pandemic. 2020. 14(4): p. 569-573.

5. Vaishya, R., et al., Artificial Intelligence (AI) applications for COVID-19 pandemic. 2020. 14(4): p. 337-339.

6. Mendhe, D., et al., Mucormycotic osteomyelitis of maxilla post-COVID patient: a case report. 2021. 39.

7. Ahmadinia, M., Ahangarikiasari, H., Data aggregation in wireless sensor networks based on environmental similarity: A learning automata approach. Journal of Networks, 2014. 9(10): p. 2567.

8. Ahmadinia, M., et al., Energy-efficient and Multi-stage Clustering Algorithm in Wireless Sensor Networks Using Cellular Learning Automata. IETE Journal of Research, 2013. 59(6): p. 774-782. 
9. Alinejad-Rokny, H., Pourshaban, H., Orimi, A. G., \& Baboli, M. M., Network motifs detection strategies and using for bioinformatic networks. Journal of Bionanoscience, 2014. 8(5): p. 353-359.

10. Bahrani, P., et al., User and item profile expansion for dealing with cold start problem. Journal of Intelligent \& Fuzzy Systems, 2020. 38(4): p. 4471-4483.

11. Mahmoudi, M.R., et al., Consensus function based on cluster-wise two level clustering. Artificial Intelligence Review, 2021. 54(1): p. 639-665.

12. Niu, H., et al., An ensemble of locally reliable cluster solutions. Applied Sciences, 2020. 10(5): p. 1891.

13. Niu, H., et al., Deep feature learnt by conventional deep neural network. Computers \& Electrical Engineering, 2020. 84: p. 106656.

14. Parvin, H., et al. A novel classifier ensemble method based on class weightening in huge dataset, in In International Symposium on Neural Networks. 2011, Springer. p. 144-150.

15. Parvin, H., et al., A classifier ensemble of binary classifier ensembles. International Journal of Learning Management Systems, 2013. 1(2): p. 37-47.

16. Parvin, H., et al., Proposing a classifier ensemble framework based on classifier selection and decision tree. Engineering Applications of Artificial Intelligence, 2015: p. 34-42.

17. Alinejad-Rokny, H., et al., Discovered motifs with using parallel Mprefixspan method. Scientific Research and Essays, 2011. 6(20): p. 4220-4226.

18. Parvin, H., et al., A heuristic scalable classifier ensemble of binary classifier ensembles. Journal of Bioinformatics and Intelligent Control, 2012. 1(2): p. 163-170.

19. Alinejad-Rokny, H., Proposing on Optimized Homolographic Motif Mining Strategy Based on Parallel Computing for Complex Biological Networks. Journal of Medical Imaging and Health Informatics, 2016. 6(2): p. 416-424.

20. Alinejad-Rokny, H., Anwar, F., Waters, S. A., Davenport, M. P., \& Ebrahimi, D., Source of CpG depletion in the HIV-1 genome. Molecular biology and evolution, 2016. 33(12): p. 32053212.

21. Alinejad-Rokny, H., Sadroddiny, E., \& Scaria, V., Machine learning and data mining techniques for medical complex data analysis. Neurocomputing, 2018. 276(1).

22. Bayati, M., et al., CANCERSIGN: a user-friendly and robust tool for identification and classification of mutational signatures and patterns in cancer genomes. Scientific reports, 2020. 10(1): p. 1-11.

23. Dashti, H., et al., Integrative analysis of mutated genes and mutational processes reveals seven colorectal cancer subtypes. bioRxiv, 2020. 2020.

24. Ghareyazi, A., et al., Whole-genome analysis of de novo somatic point mutations reveals novel mutational biomarkers in pancreatic cancer. Cancers, 2021. 13(17): p. 4376.

25. Heidari, R., et al., A systematic review of long non-coding RNAs with a potential role in Breast Cancer. Mutation Research/Reviews in Mutation Research, 2021. 787: p. 108375.

26. Javanmard, R., Jet al., Proposed a new method for rules extraction using artificial neural network and artificial immune system in cancer diagnosis. Journal of Bionanoscience, 2013. 7(6): p. 665-672. 
27. Kalantari, A., et al., Computational intelligence approaches for classification of medical data: State-of-the-art, future challenges and research directions. Neurocomputing, 2018. 276: p. 2-22.

28. Shamshirband, S., et al., A review on deep learning approaches in healthcare systems: Taxonomies, challenges, and open issues. Journal of Biomedical Informatics, 2021. 113: p. 103627.

29. Shamshirband, S., T. Rabczuk, and K.-W.J.I.A. Chau, A survey of deep learning techniques: application in wind and solar energy resources. IEEE Access 2019. 7: p. 164650-164666.

30. Khakmardan, S., et al., MHiC, an integrated user-friendly tool for the identification and visualization of significant interactions in Hi-C data. BMC genomics, 2020. 21(1): p. 1-10.

31. Rajaei, P., et al., VIRMOTIF: A user-friendly tool for viral sequence analysis. Genes, 2021. 12(2): p. 186.

32. Gooneratne, S.L., et al., Linking pig-tailed macaque major histocompatibility complex class I haplotypes and cytotoxic T lymphocyte escape mutations in simian immunodeficiency virus infection. Journal of virology, 2014. 88(24): p. 14310-14325.

33. Sharifrazi, D., et al., CNN-KCL: Automatic myocarditis diagnosis using convolutional neural network combined with k-means clustering. Preprint, 2020.

34. Aman, A.H.M., et al., IoMT amid COVID-19 pandemic: Application, architecture, technology, and security. 2020: p. 102886.

35. Bekhet, S., et al. An Artificial Intelligence Based Technique for COVID-19 Diagnosis from Chest X-Ray. in 2020 2nd Novel Intelligent and Leading Emerging Sciences Conference (NILES). 2020. IEEE.

36. Heidari, M., et al., Improving the performance of CNN to predict the likelihood of COVID-19 using chest $X$-ray images with preprocessing algorithms. 2020. 144: p. 104284.

37. Sufian, A., D.S. Jat, and A. Banerjee, Insights of artificial intelligence to stop spread of covid-19, in Big Data Analytics and Artificial Intelligence Against COVID-19: Innovation Vision and Approach. 2020, Springer. p. 177-190.

38. Guo, X., et al., A survey on machine learning in COVID-19 diagnosis. 2021.

39. Abumalloh, R.A., et al., Medical image processing and COVID-19: A literature review and bibliometric analysis. 2021.

40. Khan, M., et al., Applications of artificial intelligence in COVID-19 pandemic: A comprehensive review. 2021. 185: p. 115695.

41. Koutsos, T.M., G.C. Menexes, and C.A.J.S.o.T.T.E. Dordas, An efficient framework for conducting systematic literature reviews in agricultural sciences. 2019. 682: p. 106-117.

42. Bandyopadhyay, D. and J.J.W.p.c. Sen, Internet of things: Applications and challenges in technology and standardization. 2011. 58(1): p. 49-69.

43. Zeinab, K.A.M. and S.A.A.J.W.S.N. Elmustafa, Internet of things applications, challenges and related future technologies. 2017. 2(67): p. 126-148.

44. Keramidas, G., N. Voros, and M. Hübner, Components and Services for IoT Platforms. 2016: Springer. 
45. Adryan, B., D. Obermaier, and P. Fremantle, The technical foundations of IoT. 2017: Artech House.

46. Nagaraj, A., Introduction to Sensors in IoT and Cloud Computing Applications. 2021: Bentham Science Publishers.

47. Rahman, M.A. and A.T.J.C. Asyhari, The emergence of Internet of Things (IoT): Connecting anything, anywhere. 2019, Multidisciplinary Digital Publishing Institute. p. 40.

48. Boumehrez, F., et al., Telehealth care enhancement using the internet of things technology. 2021. 10(5): p. 2652-2660.

49. Bolhasani, H., M. Mohseni, and A.M.J.I.i.M.U. Rahmani, Deep learning applications for IoT in health care: A systematic review. 2021: p. 100550.

50. Marques, G., et al., IoT in Healthcare and Ambient Assisted Living. 2021: Springer.

51. Chamola, V., et al., A comprehensive review of the COVID-19 pandemic and the role of IoT, drones, AI, blockchain, and $5 G$ in managing its impact. 2020. 8: p. 90225-90265.

52. Taylor, W., et al., A Review of the State of the Art in Non-Contact Sensing for COVID-19. 2020. 20(19): p. 5665.

53. Khan, M.B., et al., A Systematic Review of Non-Contact Sensing for Developing a Platform to Contain COVID-19. 2020. 11(10): p. 912.

54. Mohammed, I.B. and S.M. Isa, The role of internet of things (IoT) in the containment and spread of the novel COVID-19 pandemic, in Computational Intelligence Methods in COVID19: Surveillance, Prevention, Prediction and Diagnosis. 2021, Springer. p. 109-119.

55. Roy, A., et al., Efficient Monitoring and Contact Tracing for COVID-19: A Smart IoT-Based Framework. 2020. 3(3): p. 17-23.

56. Rahman, A., et al., Adversarial examples-security threats to COVID-19 deep learning systems in medical IoT devices. 2020.

57. Ahmed, I., A. Ahmad, and G.J.I.I.o.T.J. Jeon, An IoT based deep learning framework for early assessment of Covid-19. 2020.

58. Otoom, M., et al., An IoT-based framework for early identification and monitoring of COVID-19 cases. 2020. 62: p. 102149.

59. Singh, R.P., et al., Internet of things (IoT) applications to fight against COVID-19 pandemic. 2020. 14(4): p. 521-524.

60. Vedaei, S.S., et al., COVID-SAFE: An IoT-Based System for Automated Health Monitoring and Surveillance in Post-Pandemic Life. 2020. 8: p. 188538-188551.

61. Ashraf, M.U., et al. Detection and Tracking Contagion using IoT-Edge Technologies: Confronting COVID-19 Pandemic. in 2020 International Conference on Electrical, Communication, and Computer Engineering (ICECCE). 2020. IEEE.

62. Baskaran, K., et al. IoT Based COVID Preventive System for Work Environment. in 2020 Fourth International Conference on I-SMAC (IoT in Social, Mobile, Analytics and Cloud)(I$S M A C)$. 2020. IEEE.

63. Karmore, S., et al., IoT Based Humanoid Software for Identification and Diagnosis of Covid19 Suspects. 2020. 
64. Akhund, T.M.N.U., et al. IoT Based Low-Cost Robotic Agent Design for Disabled and Covid19 Virus Affected People. in 2020 Fourth World Conference on Smart Trends in Systems, Security and Sustainability (WorldS4). 2020. IEEE.

65. Ndiaye, M., et al., IoT in the wake of COVID-19: A survey on contributions, challenges and evolution. 2020. 8: p. 186821-186839.

66. Wang, B., et al., Risk-aware identification of highly suspected covid-19 cases in social iot: A joint graph theory and reinforcement learning approach. 2020. 8: p. 115655-115661.

67. Kumar, K., N. Kumar, and R.J.I.J.o.I.N. Shah, Role of IoT to avoid spreading of COVID-19. 2020. 1: p. 32-35.

68. Kolhar, M., et al., A three layered decentralized IoT biometric architecture for city lockdown during COVID-19 outbreak. 2020. 8: p. 163608-163617.

69. De Vito, S., et al. High Resolution Air Quality Monitoring with IoT Intelligent Multisensor devices during COVID-19 Pandemic Phase 2 in Italy. in 2020 AEIT International Annual Conference (AEIT). 2020. IEEE.

70. Manalu, E.P., et al. Role Of Information Technology for Successful Responses to Covid-19 Pandemic. in 2020 International Conference on Information Management and Technology (ICIMTech). 2020. IEEE.

71. Lalmuanawma, S., et al., Applications of machine learning and artificial intelligence for Covid-19 (SARS-CoV-2) pandemic: A review. 2020: p. 110059.

72. Kyritsis, A.I. and M. Deriaz. A Queue Management Approach for Social Distancing and Contact Tracing. in 2020 Third International Conference on Artificial Intelligence for Industries (AI4I). 2020. IEEE.

73. Jordan, M.I. and T.M.J.S. Mitchell, Machine learning: Trends, perspectives, and prospects. 2015. 349(6245): p. 255-260.

74. Sra, S., S. Nowozin, and S.J. Wright, Optimization for machine learning. 2012: Mit Press.

75. Kashyap, P., Machine learning for decision makers: Cognitive computing fundamentals for better decision making. 2017: Springer.

76. Abdulkareem, K.H., et al., A review of fog computing and machine learning: concepts, applications, challenges, and open issues. 2019. 7: p. 153123-153140.

77. Shinde, P.P. and S. Shah. A review of machine learning and deep learning applications. in 2018 Fourth international conference on computing communication control and automation (ICCUBEA). 2018. IEEE.

78. Kolachalama, V.B. and P.S.J.N.d.m. Garg, Machine learning and medical education. 2018. 1(1): p. 1-3.

79. Ardabili, S., et al., Coronavirus Disease (COVID-19) Global Prediction Using Hybrid Artificial Intelligence Method of ANN Trained with Grey Wolf Optimizer. 2020.

80. Loey, M., et al., A hybrid deep transfer learning model with machine learning methods for face mask detection in the era of the COVID-19 pandemic. 2021. 167: p. 108288.

81. Singh, P. and R.J.G.t. Kaur, An integrated fog and Artificial Intelligence smart health framework to predict and prevent COVID-19. 2020. 2: p. 283-292. 
82. Vinod, D.N., S.J.C. Prabaharan, Solitons, and Fractals, Data science and the role of Artificial Intelligence in achieving the fast diagnosis of Covid-19. 2020. 140: p. 110182.

83. Karthik, R., R. Menaka, and M.J.A.S.C. Hariharan, Learning distinctive filters for COVID-19 detection from chest $X$-ray using shuffled residual CNN. 2021. 99: p. 106744.

84. Ardakani, A.A., et al., Application of deep learning technique to manage COVID-19 in routine clinical practice using CT images: Results of 10 convolutional neural networks. 2020. 121: p. 103795.

85. Polsinelli, M., L. Cinque, and G.J.P.R.L. Placidi, A light cnn for detecting covid-19 from ct scans of the chest. 2020. 140: p. 95-100.

86. Nour, M., Z. Cömert, and K.J.A.S.C. Polat, A novel medical diagnosis model for COVID-19 infection detection based on deep features and Bayesian optimization. 2020. 97: p. 106580.

87. Han, Z., et al., Accurate screening of COVID-19 using attention-based deep 3D multiple instance learning. 2020. 39(8): p. 2584-2594.

88. Marques, G., D. Agarwal, and I.J.A.S.C. de la Torre Díez, Automated medical diagnosis of COVID-19 through EfficientNet convolutional neural network. 2020. 96: p. 106691.

89. Mohammed, M.A., et al., Benchmarking methodology for selection of optimal COVID-19 diagnostic model based on entropy and TOPSIS methods. 2020. 8: p. 99115-99131.

90. Chowdhury, M.E., et al., Can AI help in screening viral and COVID-19 pneumonia? 2020. 8: p. $132665-132676$.

91. Dlamini, G.S., et al., Classification of COVID-19 and Other Pathogenic Sequences: A Dinucleotide Frequency and Machine Learning Approach. 2020. 8: p. 195263-195273.

92. Narzullaev, A., Z. Muminov, and M. Narzullaev. Contact Tracing of Infectious Diseases Using Wi-Fi Signals and Machine Learning Classification. in 2020 IEEE 2nd International Conference on Artificial Intelligence in Engineering and Technology (IICAIET). 2020. IEEE.

93. Hassan, A., I. Shahin, and M.B. Alsabek. Covid-19 detection system using recurrent neural networks. in 2020 International Conference on Communications, Computing, Cybersecurity, and Informatics (CCCI). 2020. IEEE.

94. Cihan, P. Fuzzy Rule-Based System for Predicting Daily Case in COVID-19 Outbreak. in 2020 4th International Symposium on Multidisciplinary Studies and Innovative Technologies (ISMSIT). 2020. IEEE.

95. Zheng, B., et al., MSD-Net: Multi-Scale Discriminative Network for COVID-19 Lung Infection Segmentation on CT. 2020. 8: p. 185786-185795.

96. Zheng, N., et al., Predicting COVID-19 in China using hybrid AI model. 2020. 50(7): p. 2891-2904.

97. Babukarthik, R., et al., Prediction of COVID-19 Using Genetic Deep Learning Convolutional Neural Network (GDCNN). 2020. 8: p. 177647-177666.

98. Sethi, M., et al. Sentiment identification in COVID-19 specific tweets. in 2020 International Conference on Electronics and Sustainable Communication Systems (ICESC). 2020. IEEE. 